\title{
Carthamin yellow protected rats from cerebral ischemia-reperfusion injury by improving neuronal dendritic plasticity via antiferroptosis mediated NF- KB/NLRP3 inflammasome pathway deactivation
}

\section{Hui-Hui Guo}

Nanjing University of Chinese Medicine

Li-Li Zhu

Nanjing University of Chinese Medicine

Ping-Ping Tang

Nanjing University of Chinese Medicine

Dong Chen

Nanjing University of Chinese Medicine

Yan-Cai Li

Nanjing University of Chinese Medicine

Jian-Bing Li

Nanjing University of Chinese Medicine

Chao Bao ( $\nabla$ drbaochao@163.com )

Nanjing University of Chinese Medicine https://orcid.org/0000-0002-0982-8168

\section{Research}

Keywords: ischemic stroke, Carthamin yellow, MAP-2, ferroptosis, NF-kB/NLRP3 inflammasome

Posted Date: September 9th, 2020

DOI: https://doi.org/10.21203/rs.3.rs-71460/v1

License: (c) (i) This work is licensed under a Creative Commons Attribution 4.0 International License.

Read Full License 
Carthamin yellow protected rats from cerebral ischemiareperfusion injury by improving neuronal dendritic plasticity via antiferroptosis mediated NF-кB/NLRP3 inflammasome pathway deactivation

Hui-Hui Guo ${ }^{1}$, Li-Li Zhu², Ping-Ping Tang ${ }^{2}$, Dong Chen², Yan-Cai $\mathrm{Li}^{2}$, Jian-Bing $\mathrm{Li}^{2 *}$, Chao $\mathrm{Bao}^{2^{*}}$

${ }^{1}$ Key Laboratory of Acupuncture and Medicine Research of Ministry of Education, Affiliated Hospital of Nanjing University of Chinese Medicine, Nanjing, 210023, Jiangsu, China

${ }^{2}$ Department of Acupuncture, Affiliated Hospital of Nanjing University of Chinese Medicine, Nanjing, 210029, Jiangsu, China

*Correspondence: drbaochao@163.com; njdrli@163.com 


\begin{abstract}
Background: Carthamin yellow (CY), a flavonoid compound extracted from safflower, has been reported to attenuate cardiac ischemia and reperfusion injury. It is unclear whether $\mathrm{CY}$ could ameliorate ischemic stroke.

Methods: We examined the preventive effects of CY in experimental ischemic stroke using middle cerebral artery occlusion (MCAO) rats model. Neurological function, brain edema and infarct area were assessed to elucidate the effects of $\mathrm{CY}$ on neurological function and ischemic brain injury. MAP-2 Immunofluorescence activity, expressions in NF- $\mathrm{kB} / \mathrm{NLRP} 3$ inflammasome pathway and ferroptosis were determined to reveal its underlying mechanism.
\end{abstract}

Results: A 2-week CY treatment attenuated the neurological deficit score, brain water content and infarct area in MCAO rats. Meanwhile, CY intervention increased the MAP-2 Immunofluorescence activity and deactivated NF- $\mathrm{B}$ /NLRP3 inflammasome pathway in the cortex. Declined serum TNF- $\alpha$, IL- $1 \beta$ and IL-6 concentrations were detected following $\mathrm{CY}$ administration. Furthermore, $\mathrm{CY}$ treatment inhibited $\mathrm{Fe}^{2+}$ and ROS accumulation, and restored the protein expressions of ACSL4, TFR1, GPX4 and FTH1 in the brain. The levels of GSH, SOD and MDA in the serum were reversed by CY intervention.

Conclusion: CY protected rats from ischemic stroke, which was associated with the improvement of neuronal dendritic plasticity through antiferroptosis mediated NF$\kappa \mathrm{B} / \mathrm{NLRP} 3$ inflammasome pathway deactivation.

Keywords: ischemic stroke, Carthamin yellow, MAP-2, ferroptosis, NF-кB/NLRP3 inflammasome 


\section{Introduction}

Ischemic stroke, which accounts for $85 \%$ of all stroke cases, is the leading cause of destructive cerebrovascular disease and has high mortality and morbidity rates [1]. To date, pharmacological thrombolysis is the most effective treatment which has a golden time, and only $3 \%-8 \%$ of patients are eligible for this therapy. Therefore, novel therapies against ischemic stroke are urgently required [2]. Previous studies have indicated that neuronal dendritic plasticity plays a significant role in the development of ischemic stroke [3].

Microtubule-associated protein 2 (MAP-2) is a cytoskeletal protein that is responsible for the assembly of the microtubules in the dendrites and plays a vital role in dendritic plasticity [4]. Existing evidenced showed that MAP-2 expression was decreased following ischemic cerebral injury, and enhanced neurological function was observed via MAP-2 promotion [5]. It has been proposed that the MAP-2 activity could be

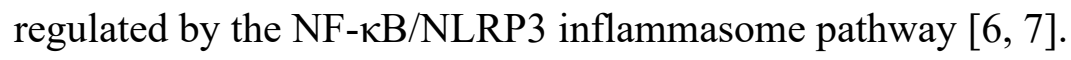

The NLRP3 inflammasome which consists of NLRP3, apoptosis-associated speck-like protein (ASC) and Caspase-1 is an important component of the innate immune system. It triggers the production of proinflammatory cytokines IL-1 $\beta$, which then facilitates the secretion of inflammatory mediators including TNF and IL-6 [8]. NF- $\kappa$ B is a transcription factor that affects the formation of NLRP3 inflammasome. The NF$\kappa \mathrm{B} /$ NLRP3 inflammasome signaling is a key mechanism of ischemic stroke. Hippocampal NF- $\kappa$ B mRNA expression and serum TNF- $\alpha$ and IL-1 $\beta$ levels were elevated in response to ischemia stroke [9]. Cerebral ischemia-reperfusion injury activated the NLRP3 inflammasome proteins in rats, while electroacupuncture mitigated NLRP3 inflammasome regulated inflammatory reaction, leading to the improvement of the neurological deficit scores and infarct sizes in MCAO rats [10]. Ferroptosis is a novel type of cell death driven by iron-dependent accumulation of lipidbased reactive oxygen species (ROS). It is regulated by the inactivation of GPX4 that can reduce lipid peroxides at the expense of GSH [11]. Numerous proteins are involved in ferroptosis including ASCL4, TFR1 and FTH1. ACSL4 is a lipid metabolism enzyme 
that is required for ferroptosis, contributing to lipid peroxidation and ferroptosis [12]. TFR1 transported iron from extracellular environment into cells, facilitating the cellular iron pool essential for ferroptosis [13]. FTH1 is a major iron storage protein responsible for maintaining the iron balance in cells [14]. Ferroptosis has been reported to influence NF- $\kappa \mathrm{B} / \mathrm{NLRP} 3$ inflammasome signaling, and is implicated in ischemic stroke $[15,16]$. Increased ROS and iron levels were noticed in the brain of ischemic stroke rodent model [17].

Carthamin yellow is a flavonoid compound isolated from safflower, an herb that is clinically used to improve blood circulation and alleviate pain, and extensively applied for the treatment of coronary heart disease, cerebrovascular disease and angiitis in China [18]. Profound antioxidant and anti-inflammatory properties have been reported in CY. CY inhibited LPS-induced activation of TNF- $\alpha$ [19]. In vivo and in vitro studies demonstrated that $\mathrm{CY}$ protected against cardiac ischemia and reperfusion injury via decreasing ROS release and NLRP3 inflammasome related inflammation response [18]. These findings raised the question whether CY could ameliorate ischemic stroke via NLRP3 inflammasome deactivation. Thus, the current study investigated the capacity of CY to prevent ischemic stroke in MCAO rats, and evaluated the possible underlying mechanism by examining changes in neuronal dendritic plasticity and NF- $\mathrm{kB} / \mathrm{NLRP} 3$ inflammasome pathway associated with ferroptosis.

\section{Materials and methods}

\section{Animals}

Male Sprague-Dawley rats, weighing 250-280 g, were purchased from Shanghai Sipper-BK laboratory animal Co. Ltd (Shanghai, China). The animals were housed in an animal center at a constant temperature of $23 \pm 1^{\circ} \mathrm{C}$, and placed on a 12-h light/dark cycle with free access to food and water. All experiments were conducted in compliance with the Provision and General Recommendation of Chinese Experimental Animals Administration Legislation. 


\section{Materials}

Carthamin yellow was obtained from Sigma-Aldrich (St. Louis, USA). All the antibodies were purchased from CST (Beverly, USA) or ProteinTech (Chicago, USA).

\section{Experimental protocol}

Animals were randomly divided into four groups including Sham, MCAO, CY $(20 \mathrm{mg} / \mathrm{kg}), \mathrm{CY}(40 \mathrm{mg} / \mathrm{kg})$. CY was administrated intragastrically to rats once daily for 2 weeks, then MCAO surgery was performed as previously described [20]. Neurological Scores, brain water content and infarct volume were determined $24 \mathrm{~h}$ after reperfusion. Immunofluorescence staining, Western blot and Flow cytometry were carried out to reveal the potential mechanisms.

\section{Neurological scoring}

For the evaluation of functional recovery, neurological score was assessed $24 \mathrm{~h}$ after MCAO by an observer blinded to the treatments as previously reported [20]. The neurological scoring ranged from 0 to 4 (normal score, 0 ; maximal deficit score, 4 ).

\section{Infarct area assessment}

Rats were sacrificed and the brains were rapidly removed. Coronal sections were cut into 2-mm slices and stained with 2\% 2,3,5-triphenyltetrazolium chloride (TTC, SigmaAldrich) solution for $30 \mathrm{~min}$ at $37^{\circ} \mathrm{C}$. Infarction size was measured by Image J software. The size of infarct regions was calculated using the following equation: \%Infarct area $=$ Infarct volume/Total volume of slice $\times 100$.

\section{Brain water content determination}

The wet-dry method was applied to determine brain oedema. The brains were immediately removed and weighed to obtain the wet weight, and were weighed again after drying in an oven at $100{ }^{\circ} \mathrm{C}$ for over $24 \mathrm{~h}$ to obtain the dry weight. The percentage of brain water content was calculated using following formula: [(wet weight) - (dry weight $)] /($ wet weight $) \times 100 \%$. 


\section{Immunofluorescence staining}

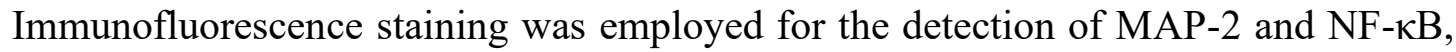
as previously described [21]. Briefly, brain tissues were obtained and fixed with $4 \%$ paraformaldehyde and transferred to $30 \%$ sucrose solution. Frozen sections, $10 \mu \mathrm{m}$ thickness, were washed with $0.01 \mathrm{M}$ PBS for $5 \mathrm{~min}$, blocked with 5\% goat serum for 1 hours at room temperature, and incubated with rabbit anti-mouse primary antibody against MAP-2 and NF- $\kappa$ B at $4{ }^{\circ} \mathrm{C}$ overnight. After washing, samples were incubated with goat anti-rabbit Alexa-fluor IgG secondary antibody for $1 \mathrm{~h}$ at room temperature. Nuclei were stained with DAPI. The stained sections were examined with a fluorescence microscope.

\section{Western blot}

Western blotting was performed as described previously [22]. Protein was isolated from samples using Trizol (Invitrogen, USA). Protein concentration was measured with the bicinchoninic acid (BCA) protein assay Kit (Thermo Scientific, USA). Equal amounts of protein were loaded on an SDS-PAGE gel and transferred to polyvinyl difluoridine (PVDF) membranes. After blocking with 5\% BSA, membranes were incubated with

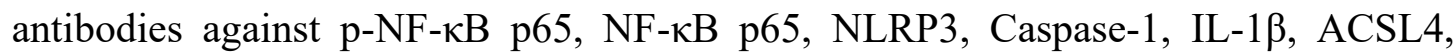
FTH1, GPX4, TFR1 and anti- $\beta$-Tublin at $4{ }^{\circ} \mathrm{C}$ overnight. After washing in TBST, immunoblots were incubated with appropriate secondary antibodies for $1 \mathrm{~h}$ at room temperature. Signals were detected using chemiluminescence and quantified by ImageJ software. Results were normalized $\beta$-Tublin expression.

\section{Lipid peroxidation measurements}

The concentrations of TNF- $\alpha$, IL-1 $\beta$, IL-6, MDA, GSH and SOD in serum were detected using different detection kits (Shanghai Beyotime Biotechnology Ltd., Shanghai, China) according to the manufacturer's instructions.

\section{ROS measurement}


ROS content was assayed by the probe of 2,7-dichlorofluorescin diacetate (DCF), a ROS detection kit (Shanghai Aladdin Bio-Chem Technology Ltd., Shanghai, China) according to the manufacturer's protocols.

\section{Iron assay}

Iron concentration was assessed employing an Iron Assay Kit (Sigma-Aldrich, St. Louis, USA), according to the manufacturer's instructions.

\section{Statistical analysis}

All statistical analyses were performed using GraphPad Prism 6 software and values are expressed as mean \pm SEM. Differences between multiple groups were analyzed by one-way ANOVA followed by Bonferroni's post hoc. Values of $p<0.05$ were considered statistically significant.

\section{Results}

\section{CY improved Neurological scores}

To determine the neuroprotective effects of CY in MCAO rats, Neurological scores were evaluated $24 \mathrm{~h}$ after reperfusion (Fig. 1a). Rats experienced MCAO modeling showed increased neurological deficit scores compared to the Sham group, while the neurological deficit was dramatically relieved after treating with CY. These results suggested that $\mathrm{CY}$ could improve neurological performance in MCAO rats.

\section{CY decreased Infarction volume and brain water content}

As shown in Fig. 1b, MCAO induced a noticeable increase in brain water content in the ischemic hemisphere at $24 \mathrm{~h}$ following reperfusion, which was remarkably reduced following CY treatment. The infarct volume at $24 \mathrm{~h}$ post-reperfusion was determined by TTC staining. TTC staining results revealed that the infarct area percentage of the brain tissue in MCAO rats was significantly higher than that in the Sham group, and lower in the $\mathrm{CY}$ administration groups in comparison with MCAO-challenged rats (Fig. 1c-d). 
These findings indicated that CY effectively attenuated cerebral infarction area and brain water content in MCAO rats.

\section{CY increased MAP-2 expression in cortex}

To examine the effects of $\mathrm{CY}$ on neuronal remodeling after MCAO challenge, Immunofluorescent staining for MAP2 (an index of dendrites) was carried out. As illustrated in Fig. 2, obvious MAP-2 immunostaining was observed in the dendrites of Sham rats. However, the MAP-2 expression in MCAO rats after 24h reperfusion declined in comparison with the Sham group. CY successfully enhanced MAP-2 expression after 14 days of drug administration. These data suggested that CY rescued the neuronal dendritic plasticity damage triggered by MCAO.

\section{CY mitigated NF-кB/NLRP3 inflammasome pathway in cortex}

We next performed Immunofluorescent staining, Western blot and ELISA to investigate the effects of $\mathrm{CY}$ on NF-kB/NLRP3 inflammasome pathway. Our results showed that in the cortex of MCAO rats, p-NF- $\mathrm{kB}$ activity in Immunofluorescent staining as well as protein levels of p-NF- $\kappa \mathrm{B}, \mathrm{NLRP} 3$, Caspase-1 and IL-1 $\beta$ in Western blot were markedly increased compared with the Sham group, whereas CY administration significantly reversed this increase in MCAO rats (Fig. 3-4). In addition, MCAO group showed evident higher levels of TNF- $\alpha$, IL-1 $\beta$, IL-6 in the serum (Fig. 5). Strikingly, upon CY treatment, a remarkable decrease in TNF- $\alpha$, IL-1 $\beta$, IL-6 were observed. Our findings showed that $\mathrm{CY}$ treatment inhibited the NF-kB/NLRP3 inflammasome pathway in rats that underwent MCAO modeling.

\section{CY attenuated ferroptosis}

To determine the involvement of ferroptosis in MCAO rats, we detected iron and ROS accumulation, lipid peroxidation level, and the expressions of ferroptosis-related proteins. As was depicted in Fig. 6, rats experienced MCAO challenge was associated with obvious ROS and iron accumulation in the cortex relative to the Sham rats. In addition, increased MDA generation along with reduced GSH and SOD levels in the 
serum were also observed after MCAO modeling (Fig. 7). Western blot assay showed MCAO challenge dramatically promoted the protein levels of ACSL4 and TFR1 in the cortex, while decreased the protein expressions of FTH1 and GPX4 (Fig. 8). It is noteworthy that the above changes were significantly ameliorated via treatment with $\mathrm{CY}$, indicating the anti-ferroptosis efficacy of CY in MCAO rats.

\section{Discussion}

In the present study, effect of CY in the development of ischemic stroke in animals was invested. It was found that CY improved the neurological deficit score, brain water content and infarct area in MCAO rats. CY also enhanced MAP-2 expression and deactivated NF-kB/NLRP3 inflammasome pathway in cortex. Decreased serum concentrations of TNF- $\alpha$, IL- $1 \beta$ and IL- 6 were observed following CY interventions. Furthermore, $\mathrm{CY}$ alleviated MCAO elicited ferroptosis, evidenced by reduced iron and ROS accumulation, declined lipid peroxidation level and restored ferroptosis-related protein expressions.

Middle cerebral artery occlusion (MCAO) rodent model is the most frequently used animal model of ischemic stroke, which is valuable in exploring the etiology of the disease and evaluating the effects of treatment strategies. Animals experienced cerebral ischemia and reperfusion have been shown to have neurological movement disorders and pathological changes including brain edema and infarct area [23]. In our study, elevated neurological deficit score, brain water content and cerebra infarct area were detected after MCAO challenge, suggesting the successful induction of ischemic stroke in rats. However, CY alleviated the neurological deficits, brain edema and infarct area resulted from $\mathrm{MCAO}$, indicating that $\mathrm{CY}$ pretreatment was effective in improving neurological function and ischemic brain injury in MCAO rats.

Dendrites are a major determinant of how neurons integrate and process incoming information. The plasticity of dendrites plays a vital role in the functional properties of neural circuits [24]. Aberrant dendritic plasticity was involved in the progress of ischemic stroke. A study monitored the changes of dendritic plasticity in ischemic 
stroke using two-photon microscopy, which showed that while experiencing ischemic stroke, the dendrites disintegrated within minutes of ischemia; increased dendritic spine turnover was observed in the surviving peri-infarct zone many weeks after stroke [3]. Lin explored the correlation between postsynaptic density-95 (PSD95) gene 3'UTR single nucleotide polymorphism (SNP) and the risk of ischemic stroke, which revealed that PSD95 gene SNPs are associated with the occurrence and prognosis of ischemic stroke [25].

MAP-2, a protein that was enriched in neuronal dendrites, regulates microtubule dynamics in dendrites and is important for dendritic plasticity. MAP-2 loss has been documented after cerebral ischemic, and therapies that enhanced MAP-2 expression decreased the mortality and neurological impairments of MCAO rats [26]. In our study, declined MAP-2 Immunofluorescence activity was noticed in rats subjected to MCAO modeling, while CY inhibited this decrease and improved the neurological outcomes and pathological changes in MCAO animals, reflecting the essential role of MAP-2 in experimental ischemic stroke and CY-generated therapeutic efficacy.

$\mathrm{NF}-\kappa \mathrm{B}$ is a pivotal regulator of inflammation-related gene transcription. Studies have shown that NF- $\kappa \mathrm{B}$ could serve as an upstream activator of NLRP3 inflammasome, which is consisted of NLRP3, ASC and caspase-1 and contributed to the production of TNF- $\alpha$, IL-1 $\beta$ and IL-6. The NF- $\mathrm{B}$ /NLRP3 inflammasome pathway has been reported to be closely related to the regulation of MAP-2 activity. Reduced MAP-2

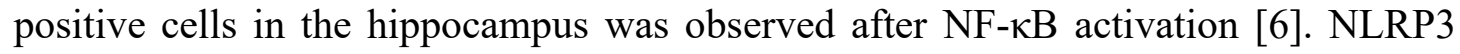
inflammasome led to the loss of MAP2-immunoreactive dendrites in the hippocampus CA1 region, which was greatly reversed by NLRP3 and caspase-1 inhibitors [7]. The NF- $\kappa$ B/NLRP3 inflammasome pathway has been implicated in ischemic stroke. Terai et al. examined the distribution of NF- $\mathrm{kB}$ on the brain samples obtained from patients who died after stroke, and discovered that the immunoreactivity for NF- $\mathrm{BB}$ was enhanced in glial cells of infarcted areas in comparison with control cases [27]. Arctigenin, a phenylpropanoid dibenzylbutyrolactone lignan derived from Arctium lappa $\mathrm{L}$, has been uncovered to avoid MCAO-induced neuronal deterioration, cerebral infarct and brain water content by inhibiting NLRP3 inflammasome activation and IL- 
$1 \beta$ secretion [28]. We found that the expression of NF- $\mathrm{B}$ /NLRP3 inflammasome signaling was upregulated in the cortex of MCAO rats, accompanied by elevated levels of TNF- $\alpha$, IL-1 $\beta$, IL- 6 in the serum; Upon CY administration, the above changes were restored, confirming the involvement of NF- $\mathrm{KB} / \mathrm{NLRP} 3$ inflammasome signaling in the pathology and treatment of ischemic stroke.

Ferroptosis is a newly defined cell death process characterized by the iron-dependent accumulation of lipid peroxides to lethal levels. Increased protein levels in NF-

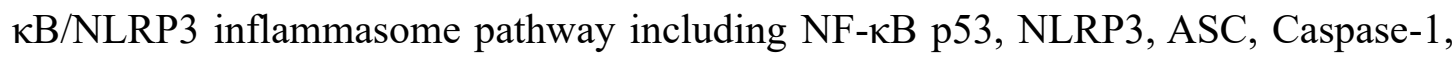
IL-1 $\beta$ has been detected in ferroptosis [15]. The inflammatory cytokine levels such as IL-1 $\beta$, IL-6 and TNF- $\alpha$ were profoundly decreased post ferroptosis inhibitor treatment [29]. An increasing amount of literature has highlighted the role of ferroptosis in ischemic stroke. The content of free iron and MDA in brain tissues were significantly augmented while the levels of GPX4 and GSH were greatly declined in animals experienced MCAO [30]. Tau ablation prevented mice from MCAO induced iron accumulation in the brain and avoided ferroptosis, leading to the attenuation of the ischemia-reperfusion injury including neurological defects, infarct area, motor and cognitive dysfunctions [31]. We observed that MCAO challenge promoted cortex iron and ROS accumulation and serum lipid peroxidation level, resulted in aberrant expressions of ferroptosis-related proteins in the brain, whereas treatment with $\mathrm{CY}$ inhibited these changes, suggesting that ferroptosis might partially account for experimental ischemic stroke progression and CY-related treatment effects on MCAO rats.

\section{Conclusion}

Taken together, we demonstrated that $\mathrm{CY}$ prevented rats from ischemia-reperfusion injury via neuronal dendritic plasticity enhancement by antiferroptosis mediated NFผB/NLRP3 inflammasome deactivation. Our results suggest the possibility of using CY as a therapeutic agent for ischemic stroke treatment. 


\section{Ethics approval and consent to participate}

Not applicable.

\section{Consent for publication}

Not applicable.

\section{Availability of data and materials}

The data used to support the findings of this study are available from the corresponding author upon request.

\section{Competing interests}

The authors declare that they have no competing interests.

\section{Funding}

This work was supported by the Science and Technology Project of Jiangsu Provincial Bureau of Traditional Chinese Medicine (JD201812) and the Social Development Project of Jiangsu Science and Technology Department (BE2017769).

\section{Authors' contributions}

HG, CB and JL were involved in design of the study. HG, LZ and PT performed the experiments. DC analyzed the data. YL drafted the manuscript with the support of all authors.

\section{Acknowledgements}

Not applicable. 


\section{References}

1. G. Cao, N. Jiang, Y. Hu, et al. "Ruscogenin Attenuates Cerebral Ischemia-Induced Blood-Brain Barrier Dysfunction by Suppressing TXNIP/NLRP3 Inflammasome Activation and the MAPK Pathway," Int J Mol Sci, vol. 17, no. 9.

2. P.W. So, A. Ekonomou, K. Galley, et al. "Intraperitoneal delivery of acetate-encapsulated liposomal nanoparticles for neuroprotection of the penumbra in a rat model of ischemic stroke," Int J Nanomedicine, vol. 14, pp. 1979-1991.

3. C.E. Brown and T.H. Murphy. "Livin' on the edge: imaging dendritic spine turnover in the periinfarct zone during ischemic stroke and recovery," Neuroscientist, vol. 14, no. 2, pp. 139-146.

4. J. Raber, E.R.S. Torres, T. Akinyeke, et al. "Detrimental Effects of Helium Ion Irradiation on Cognitive Performance and Cortical Levels of MAP-2 in B6D2F1 Mice," Int J Mol Sci, vol. 19, no. 4.

5. J.B. Lin, C.J. Zheng, X. Zhang, J. Chen, W.J. Liao, and Q. Wan. "Effects of Tetramethylpyrazine on Functional Recovery and Neuronal Dendritic Plasticity after Experimental Stroke," Evid Based Complement Alternat Med, vol. 2015, p. 394926.

6. J. Zhao, W. Bi, S. Xiao, et al. "Neuroinflammation induced by lipopolysaccharide causes cognitive impairment in mice," Sci Rep, vol. 9, no. 1, p. 5790.

7. Y. Chang, J. Zhu, D. Wang, et al. "NLRP3 inflammasome-mediated microglial pyroptosis is critically involved in the development of post-cardiac arrest brain injury," J Neuroinflammation, vol. 17, no. 1, p. 219.

8. A.A. Negash, R.M. Olson, S. Griffin, and M. Gale, Jr. "Modulation of calcium signaling pathway by hepatitis C virus core protein stimulates NLRP3 inflammasome activation," PLoS Pathog, vol. 15, no. 2, p. e1007593.

9. H. Wu, C. Tang, L.W. Tai, et al. "Flurbiprofen axetil attenuates cerebral ischemia/reperfusion injury by reducing inflammation in a rat model of transient global cerebral ischemia/reperfusion," Biosci Rep, vol. 38 , no. 4 .

10. T. Jiang, M. Wu, Z. Zhang, et al. "Electroacupuncture attenuated cerebral ischemic injury and neuroinflammation through alpha7nAChR-mediated inhibition of NLRP3 inflammasome in stroke rats," Mol Med, vol. 25, no. 1, p. 22.

11. L. Wang, Y. Liu, T. Du, et al. "ATF3 promotes erastin-induced ferroptosis by suppressing system Xc(.)," Cell Death Differ, vol. 27, no. 2, pp. 662-675.

12. G. Lei, Y. Zhang, P. Koppula, et al. "The role of ferroptosis in ionizing radiation-induced cell death and tumor suppression," Cell Res, vol. 30, no. 2, pp. 146-162.

13. H. Feng, K. Schorpp, J. Jin, et al. "Transferrin Receptor Is a Specific Ferroptosis Marker," Cell Rep, vol. 30, no. 10, pp. 3411-3423 e3417.

14. J. Lu, X. Liu, Y. Tian, et al. "Moxibustion Exerts a Neuroprotective Effect through Antiferroptosis in Parkinson's Disease," Evid Based Complement Alternat Med, vol. 2019, p. 2735492.

15. J. Wang, B. Deng, Q. Liu, et al. "Pyroptosis and ferroptosis induced by mixed lineage kinase 3 (MLK3) signaling in cardiomyocytes are essential for myocardial fibrosis in response to pressure overload," Cell Death Dis, vol. 11, no. 7, p. 574.

16. Y. Zhang, C. Sun, C. Zhao, et al. "Ferroptosis inhibitor SRS 16-86 attenuates ferroptosis and promotes functional recovery in contusion spinal cord injury," Brain Res, vol. 1706, pp. 48-57.

17. X. Guan, X. Li, X. Yang, et al. "The neuroprotective effects of carvacrol on ischemia/reperfusioninduced hippocampal neuronal impairment by ferroptosis mitigation," Life Sci, vol. 235, p. 116795. 
18. Q.Y. Lu, J.Q. Ma, Y.Y. Duan, et al. "Carthamin Yellow Protects the Heart Against Ischemia/Reperfusion Injury With Reduced Reactive Oxygen Species Release and Inflammatory Response," J Cardiovasc Pharmacol, vol. 74, no. 3, pp. 228-234.

19. B. Chen, H.T. Wang, B. Yu, J.D. Zhang, and Y. Feng. "Carthamin yellow inhibits matrix degradation and inflammation induced by LPS in the intervertebral disc via suppression of MAPK pathway activation," Exp Ther Med, vol. 14, no. 2, pp. 1614-1620.

20. R. Sun, Y. Song, S. Li, et al. "Levo-tetrahydropalmatine Attenuates Neuron Apoptosis Induced by Cerebral Ischemia-Reperfusion Injury: Involvement of c-Abl Activation," J Mol Neurosci, vol. 65, no. 3, pp. 391-399.

21. Y. Yang, Z. Hu, X. Du, H. Davies, X. Huo, and M. Fang. "miR-16 and Fluoxetine Both Reverse Autophagic and Apoptotic Change in Chronic Unpredictable Mild Stress Model Rats," Front Neurosci, vol. 11, p. 428.

22. S. Chen, Z. Dong, M. Cheng, et al. "Homocysteine exaggerates microglia activation and neuroinflammation through microglia localized STAT3 overactivation following ischemic stroke," $J$ Neuroinflammation, vol. 14, no. 1, p. 187.

23. P. Guo, Z. Jin, H. Wu, et al. "Effects of irisin on the dysfunction of blood-brain barrier in rats after focal cerebral ischemia/reperfusion," Brain Behav, vol. 9, no. 10, p. e01425.

24. P.W. Hickmott and I.M. Ethell. "Dendritic plasticity in the adult neocortex," Neuroscientist, vol. 12, no. 1, pp. 16-28.

25. Y.Y. Lin, T.Y. Yu, H. Quan, Y.J. Chen, X.Y. Liu, and D.Y. Huang. "Association Between PSD95 Gene 3'UTR Single Nucleotide Polymorphism and Risk of Acute Ischemic Stroke in Chinese Han Population," J Mol Neurosci, vol. 70, no. 9, pp. 1389-1402.

26. R. Pan, J. Cai, L. Zhan, et al. "Buyang Huanwu decoction facilitates neurorehabilitation through an improvement of synaptic plasticity in cerebral ischemic rats," BMC Complement Altern Med, vol. 17, no. 1, p. 173.

27. K. Terai, A. Matsuo, E.G. McGeer, and P.L. McGeer. "Enhancement of immunoreactivity for NFkappa B in human cerebral infarctions," Brain Res, vol. 739, no. 1-2, pp. 343-349.

28. S. Zhang, L. Jiang, F. Che, Y. Lu, Z. Xie, and H. Wang. "Arctigenin attenuates ischemic stroke via SIRT1-dependent inhibition of NLRP3 inflammasome," Biochem Biophys Res Commun, vol. 493, no. 1, pp. 821-826.

29. Y. Sun, P. Chen, B. Zhai, et al. "The emerging role of ferroptosis in inflammation," Biomed Pharmacother, vol. 127, p. 110108.

30. J. Lu, F. Xu, and H. Lu. "LncRNA PVT1 regulates ferroptosis through miR-214-mediated TFR1 and p53," Life Sci, p. 118305.

31. Q.Z. Tuo, P. Lei, K.A. Jackman, et al. "Tau-mediated iron export prevents ferroptotic damage after ischemic stroke," Mol Psychiatry, vol. 22, no. 11, pp. 1520-1530. 


\section{Figure legends}

Fig. 1 Effects of $\mathrm{CY}$ on neurological deficit score, brain water content and infarct area. (a) Neurological deficit score, (b) Brain water content, (c-d) Infarct area. Data are presented as mean \pm SEM. ${ }^{\#} p<0.01$ vs Sham; ${ }^{*} p<0.05,{ }^{* *} p<0.01$ vs MCAO.

Fig. 2 MAP-2 expression in cortex of MCAO rats. (a) Representative image of Immunofluorescence staining for MAP-2, (b) Quantification analysis of MAP-2. Data are presented as mean \pm SEM. \#\# $p<0.01$ vs Sham; $* p<0.05$ vs MCAO.

Fig. 3 p-NF- $\mathrm{kB}$ expression in cortex of MCAO rats. (a) Representative image of Immunofluorescence staining for $\mathrm{p}-\mathrm{NF}-\mathrm{\kappa B}$, (b) Quantification analysis of $\mathrm{p}-\mathrm{NF}-\kappa \mathrm{B}$. Data are presented as mean \pm SEM. ${ }^{\#} p<0.01$ vs Sham; $* p<0.05$ vs MCAO.

Fig. 4 Effects of $\mathrm{CY}$ on NF-kB/NLRP3 inflammasome pathway in cortex. (a) Representative immunoblots, (b-e) Quantification of the relative protein levels. Data are presented as mean $\pm \mathrm{SEM}$. \# $p<0.01$ vs Sham; $* p<0.05,{ }^{* *} p<0.01$ vs MCAO.

Fig. 5 TNF- $\alpha$, IL-1 $\beta$ and IL-6 concentrations in serum. (a) TNF- $\alpha$, (b) IL-1 $\beta$, (c) IL-6. Data are presented as mean \pm SEM. ${ }^{\#} p<0.01$ vs Sham; $* p<0.05, * * p<0.01$ vs MCAO.

Fig. 6 ROS and iron accumulation in cortex. (a-b) ROS generation, (c) $\mathrm{Fe}^{2+}$ production. Data are presented as mean \pm SEM. ${ }^{\#} p<0.01$ vs Sham; $* * p<0.01$ vs MCAO.

Fig. 7 lipid peroxidation level in serum. (a) GSH, (b) SOD, (c) MDA. Data are presented as mean \pm SEM. ${ }^{\#} p<0.01$ vs Sham; $* p<0.05,{ }^{* *} p<0.01$ vs MCAO.

Fig. 8 Expression levels of ferroptosis-related proteins in cortex. (a) Representative immunoblots, (b-e) Quantification of the relative protein levels. Data are presented as mean \pm SEM. ${ }^{\#} p<0.05$, ${ }^{\#}$ $p<0.01$ vs Sham; $* p<0.05, * * p<0.01$ vs MCAO. 
Figures

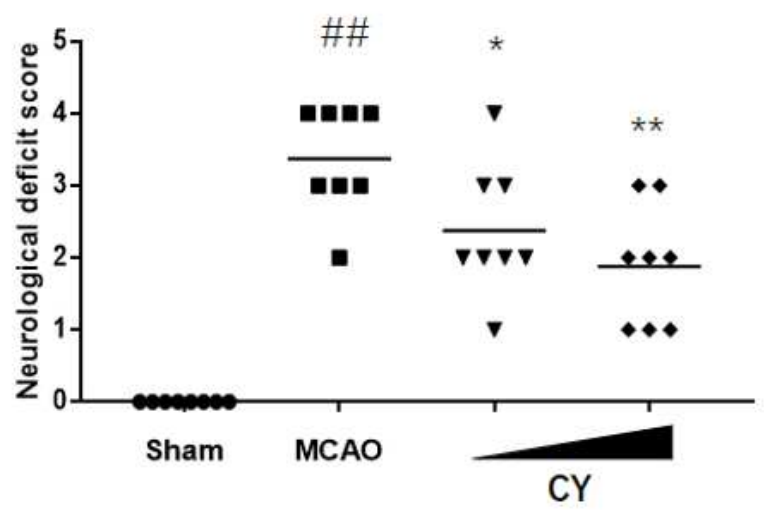

C

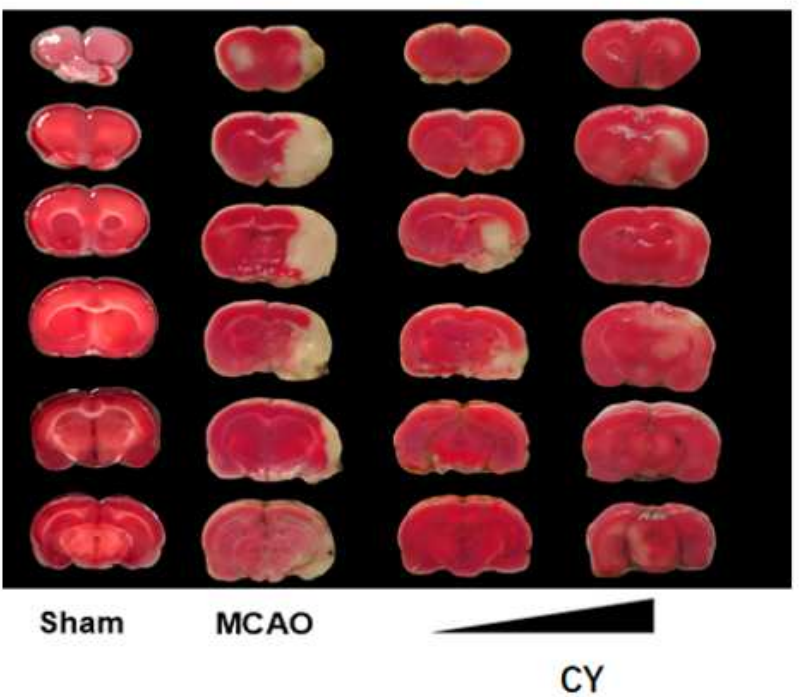

b

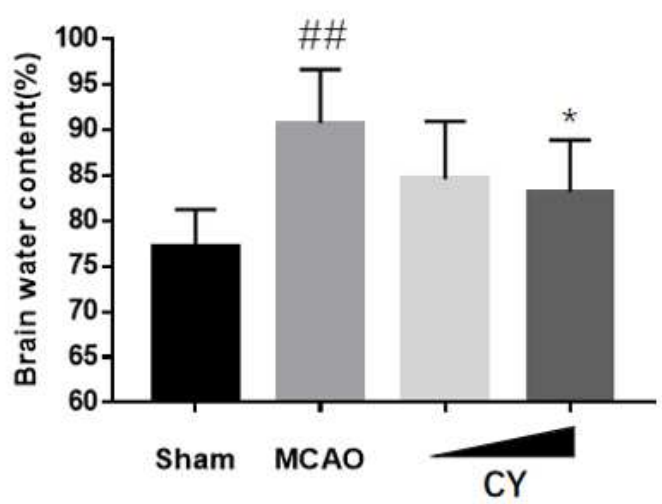

d

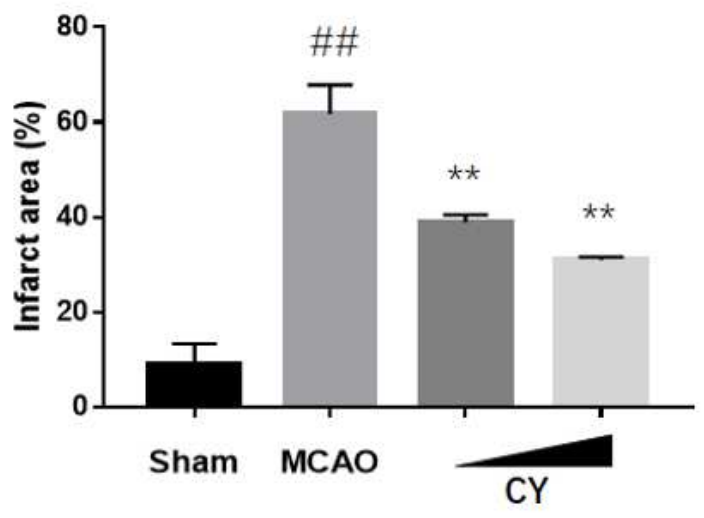

Figure 1

Effects of CY on neurological deficit score, brain water content and infarct area. (a) Neurological deficit score, (b) Brain water content, (c-d) Infarct area. Data are presented as mean \pm SEM. \#\# p $<0.01$ vs Sham; * $p<0.05, * * p<0.01$ vs MCAO. 
a
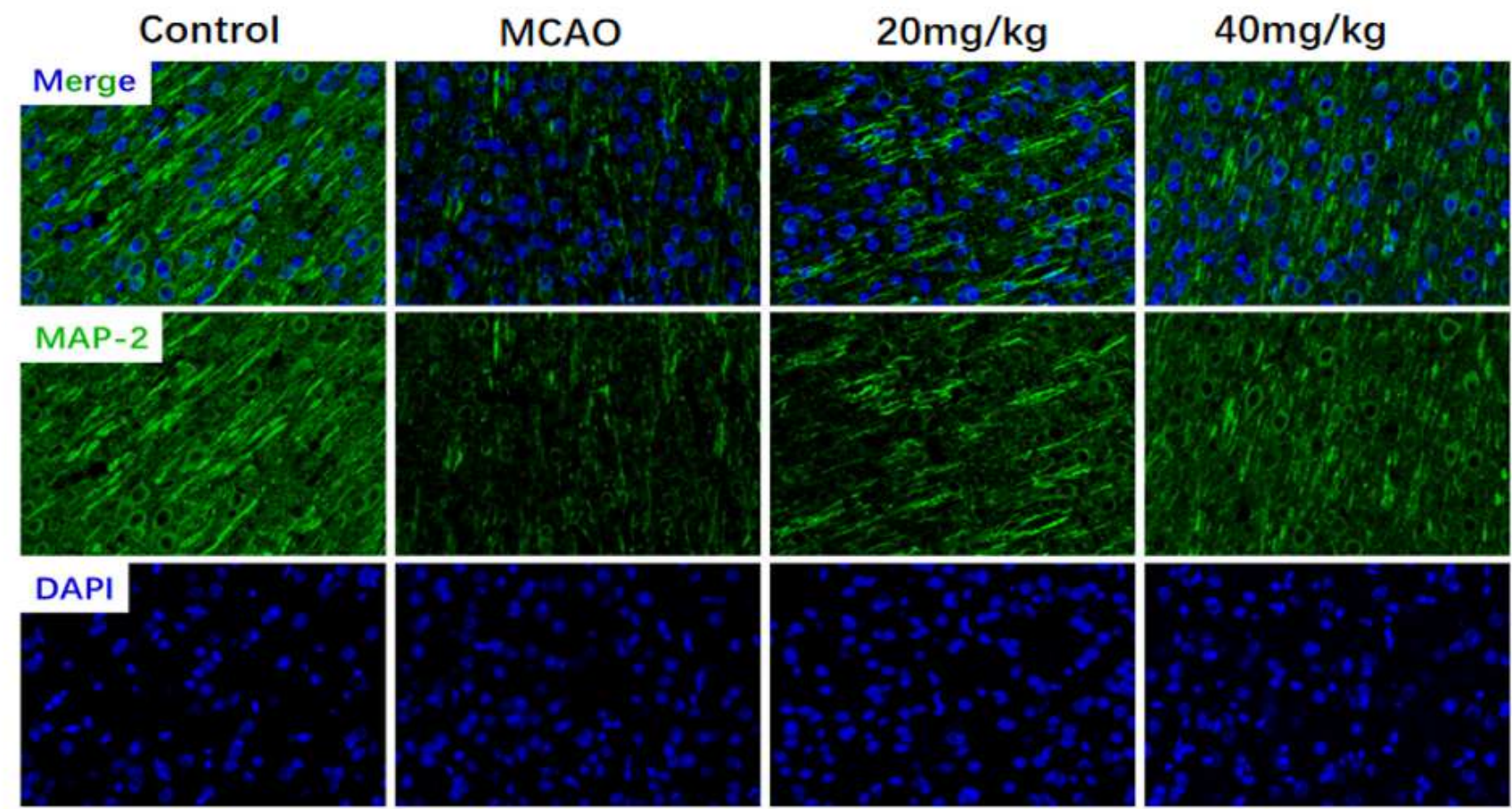

b
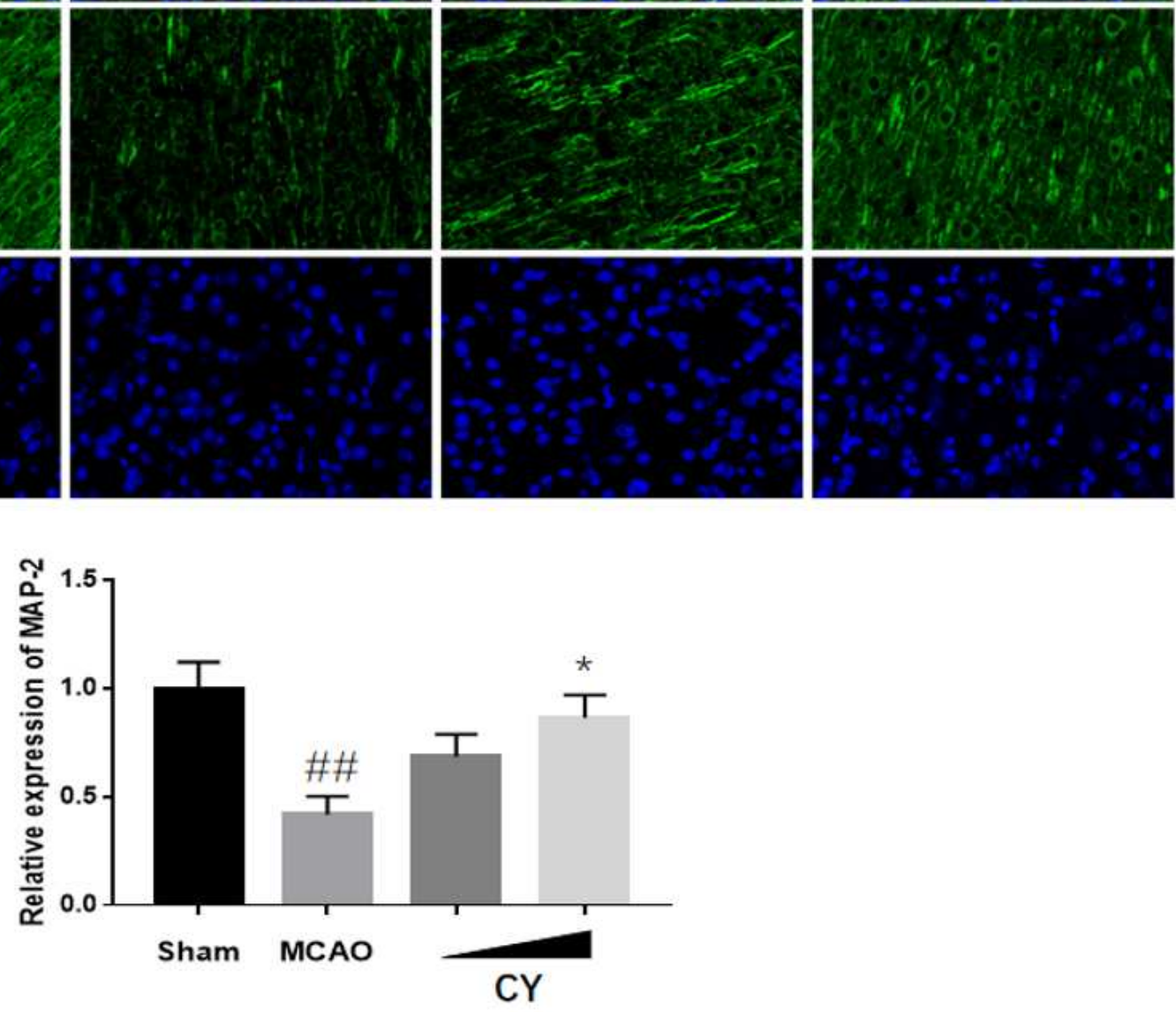

Figure 2

MAP-2 expression in cortex of MCAO rats. (a) Representative image of Immunofluorescence staining for MAP-2, (b) Quantification analysis of MAP-2. Data are presented as mean \pm SEM. \#\# $p<0.01$ vs Sham; * $p<0.05$ vs MCAO. 
a

Control

MCAO

$20 \mathrm{mg} / \mathrm{kg}$

$40 \mathrm{mg} / \mathrm{kg}$
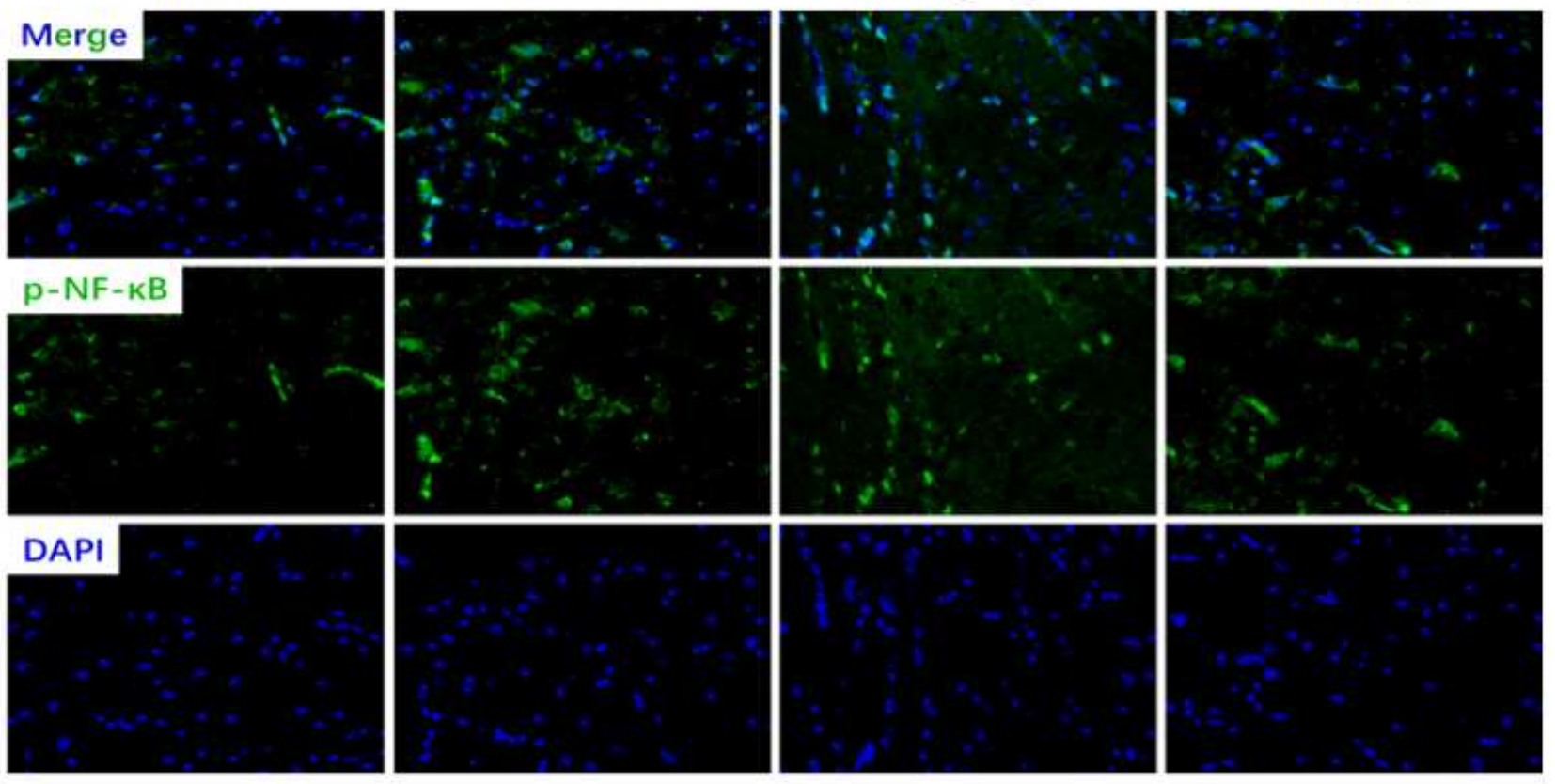

b

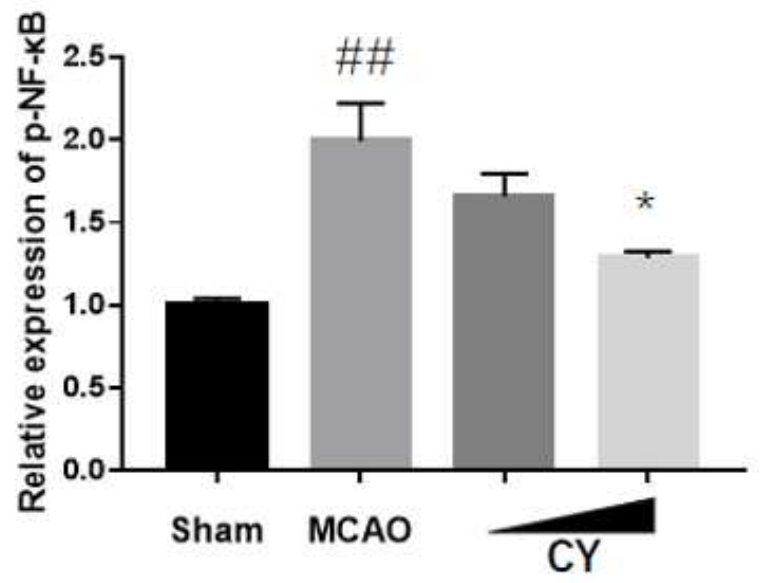

Figure 3

p-NF-KB expression in cortex of MCAO rats. (a) Representative image of Immunofluorescence staining for p-NF-KB, (b) Quantification analysis of p-NF-KB. Data are presented as mean \pm SEM. \#\# $p<0.01$ vs Sham; $* p<0.05$ vs MCAO. 
a

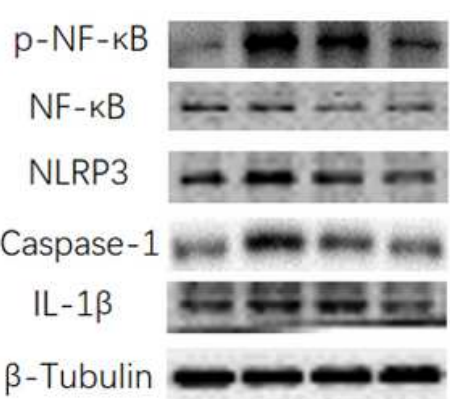

b

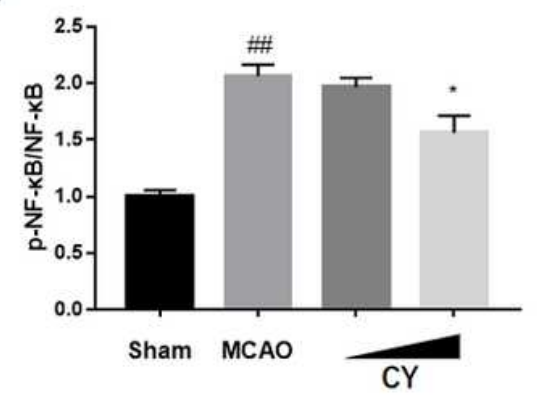

d

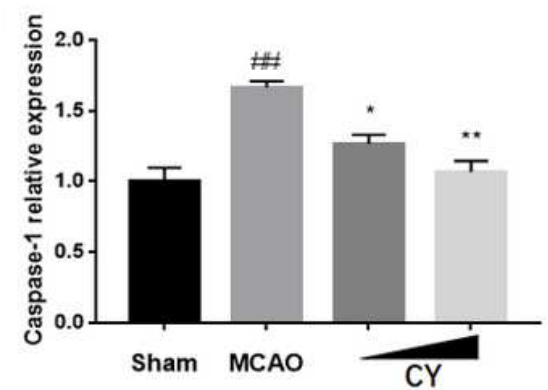

c

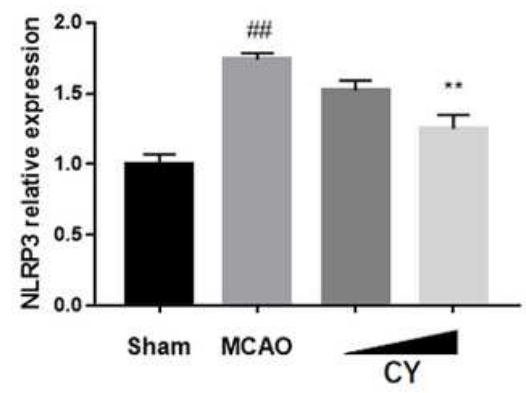

e

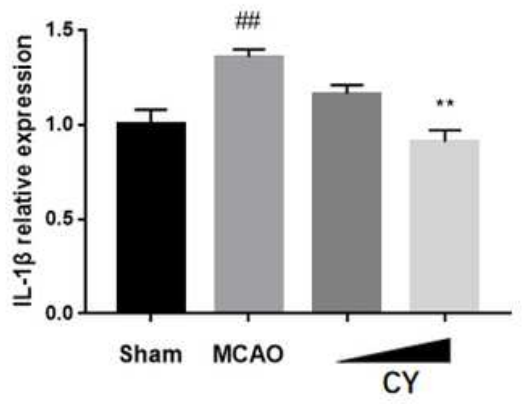

\section{Figure 4}

Effects of CY on NF-KB/NLRP3 inflammasome pathway in cortex. (a) Representative immunoblots, (b-e) Quantification of the relative protein levels. Data are presented as mean \pm SEM. \#\# $p<0.01$ vs Sham; * $p$ $<0.05$, ** $p<0.01$ vs MCAO.

a

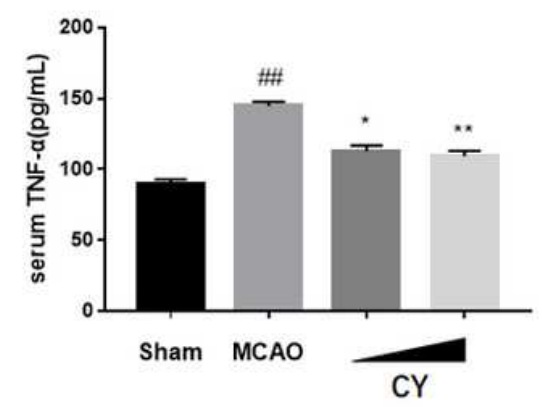

b

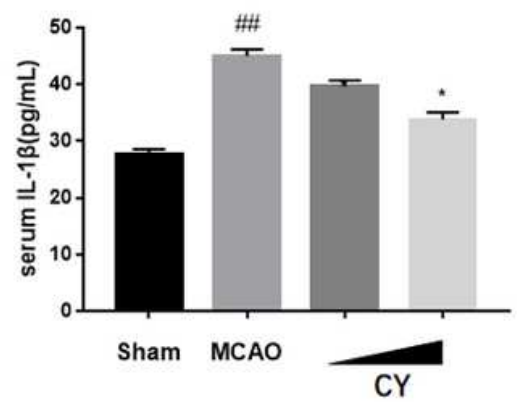

C

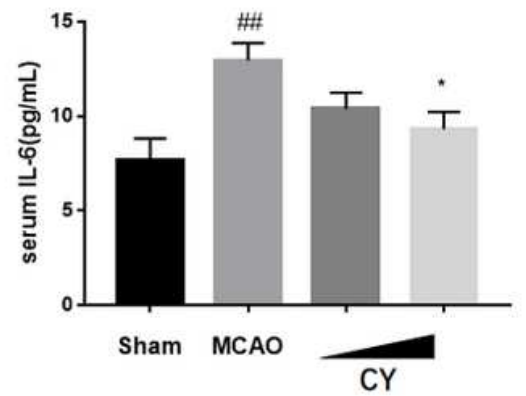

Figure 5

TNF- $\alpha$, IL-1 $\beta$ and IL- 6 concentrations in serum. (a) TNF-a, (b) IL-1 $\beta$, (c) IL-6. Data are presented as mean \pm SEM. \#\# $p<0.01$ vs Sham; * $p<0.05$, ** $p<0.01$ vs MCAO. 
a
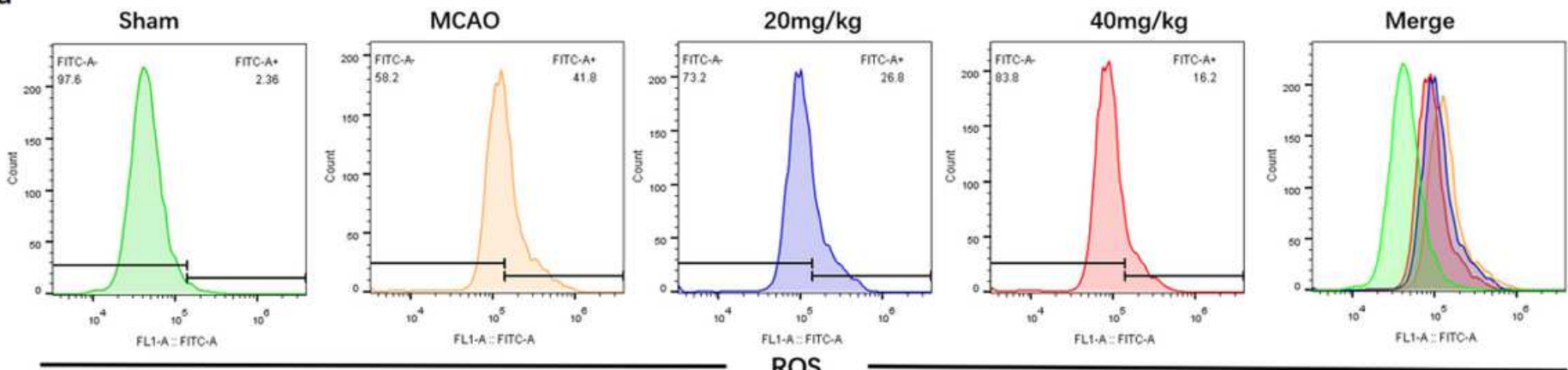

b

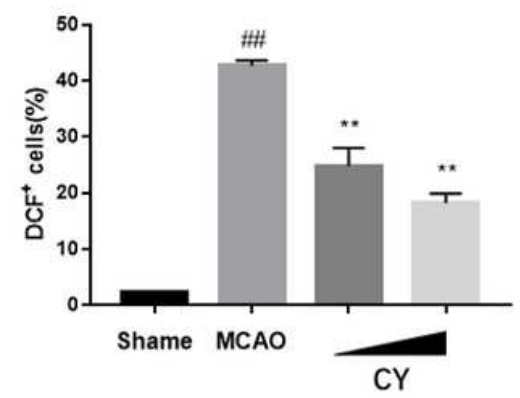

C

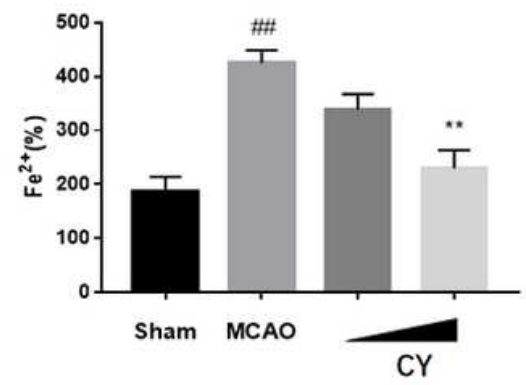

Figure 6

ROS and iron accumulation in cortex. (a-b) ROS generation, (c) Fe2+ production. Data are presented as mean \pm SEM. \#\# $p<0.01$ vs Sham; ** $p<0.01$ vs MCAO.

a

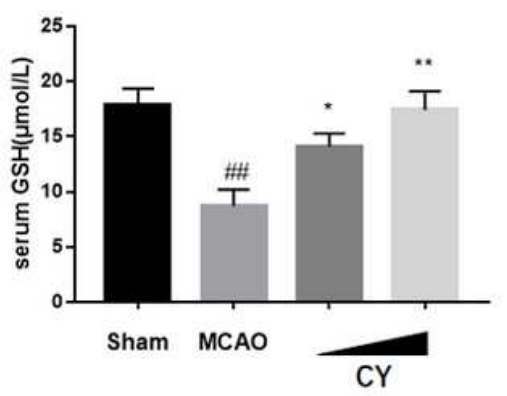

b

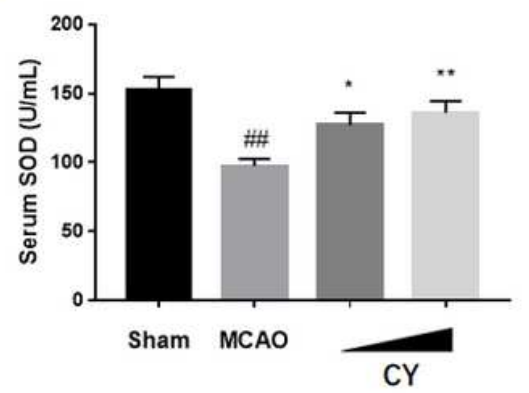

C

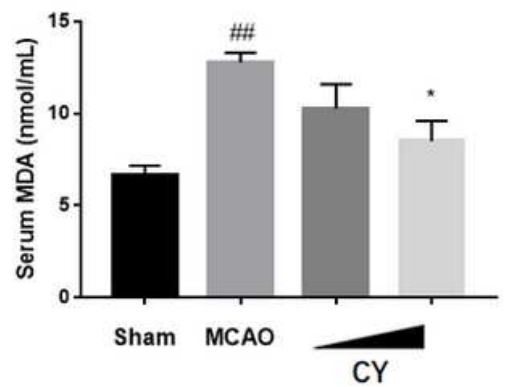

Figure 7

lipid peroxidation level in serum. (a) GSH, (b) SOD, (c) MDA. Data are presented as mean \pm SEM. \#\# $p<$ 0.01 vs Sham; * $p<0.05$, ** $p<0.01$ vs MCAO. 
a

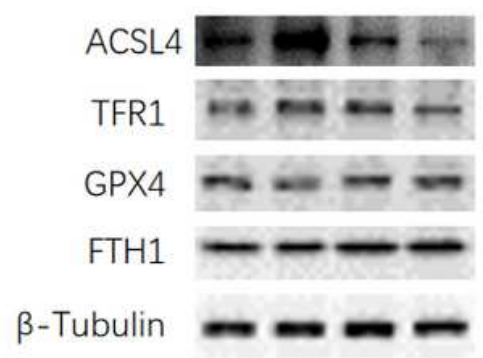

b

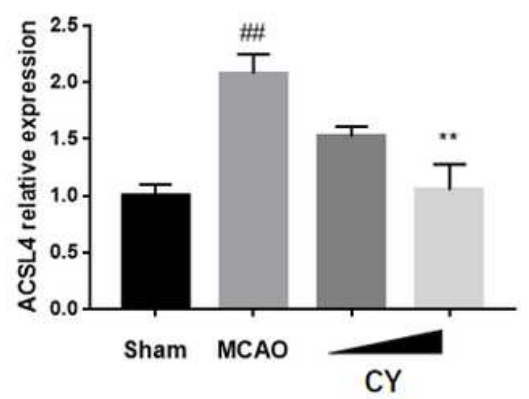

d

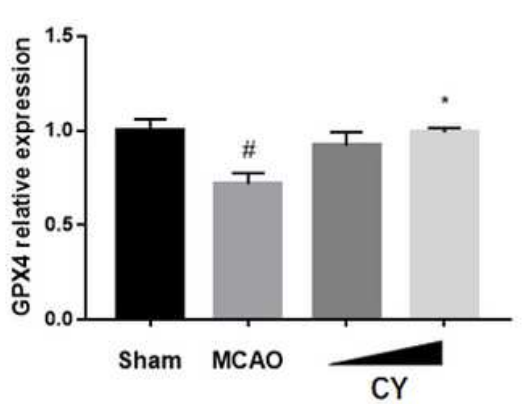

c

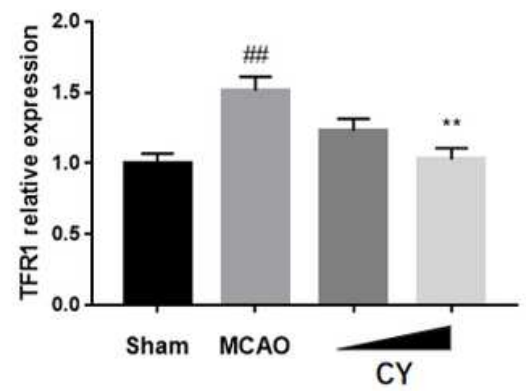

e

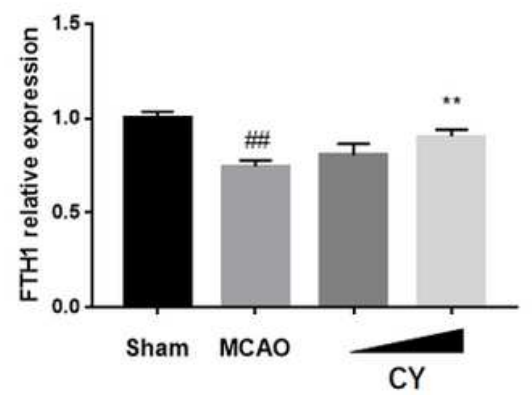

Figure 8

Expression levels of ferroptosis-related proteins in cortex. (a) Representative immunoblots, (b-e) Quantification of the relative protein levels. Data are presented as mean \pm SEM. $\# p<0.05, \# \# p<0.01$ vs Sham; ${ }^{*} p<0.05, * * p<0.01$ vs MCAO. 